TRANSACTIONS OF THE

AMERICAN MATHEMATICAL SOCIETY

Volume 211, 1975

\title{
ON SUBNORMAL OPERATORS
}

BY

\section{RADJABALIPOUR}

ABSTRACT. Let $T$ be the adjoint of a subnormal operator defined on a Hilbert space $H$. For any closed set $\delta$, let $X_{T}(\delta)=\{x \in H$ : there exists an analytic function $f_{x}: C \backslash \delta \rightarrow H$ such that $\left.(z-T) f_{x}(z) \equiv x\right\}$. It is shown that $T$ is decomposable (resp. normal) if $X_{T}\left(\partial G_{a}\right)$ is closed (resp. if $\left.X_{T}\left(\partial G_{a}\right)=\{0\}\right)$ for a certain family $\left\{G_{a}\right\}$ of open sets. Some of the results are extended to the case that $T$ is the adjoint of the restriction of a spectral or decomposable operator to an invariant subspace.

Putnam [17] and Stampfli [20] approach the invariant subspace problem for a hyponormal (cohyponormal) operator $T$ by studying the analytic continuability of the local resolvents $(z-T)^{-1} x$ for individual vectors $x$ in the underlying Hilbert space. Here, by independent proofs, we find some necessary and sufficient conditions for normality or decomposability of a subnormal (cosubnormal) operator in terms of its local resolvents.

1. Preliminaries. Let $B(H)$ denote the algebra of all bounded linear operators defined on a Hilbert space $H$. We recall the following definitions and facts about the elements of $B(H)$.

(i) An operator $T \in B(H)$ is called spectral if $T=S+Q$ where $S$ is similar to a normal operator, $Q$ is a quasinilpotent operator, and $S Q=Q S$ [8, pp. 1939 and 1947]. Moreover $T$ has a (not necessarily orthogonal) resolution of the identity which coincides with that of $S$.

(ii) The restriction of a normal (resp. spectral) operator to an invariant subspace is called a subnormal (resp. subspectral) operator; the adjoint of a subnormal (resp. subspectral) operator is called a cosubnormal (resp. cosubspectral) operator.

(iii) An operator $T \in B(H)$ is hyponormal if $T^{*} T-T T^{*} \geq 0$ and cohyponormal if $T^{*} T-T T^{*} \leq 0$.

Received by the editors November 11, 1974.

AMS (MOS) subject classifications (1970). Primary 47B40.

Key words and phrases. Hilbert space, normal operator, spectral operator, subnormal operator, decomposable operator, spectral subspace.

Copyright $\odot$ 1975, American Mathematical Society 
(iv) Every subnormal operator is hyponormal.

(v) For an operator $T \in B(H)$ and a closed subset $\delta$ of the complex plane $C$ we define

$$
\begin{aligned}
& X_{T}(\delta)=\{x \in H: \text { there exists an analytic function } \\
& \left.\qquad f_{x}: \mathbf{C} \backslash \delta \rightarrow H \text { such that }(z-T) f_{x}(z) \equiv x\right\} .
\end{aligned}
$$

The set $X_{T}(\delta)$ is a hyperinvariant linear manifold of $T$. If $\delta$ and $\gamma$ are two disjoint closed subsets of $\mathbf{C}$, then

$$
X_{T}(\delta) \cap X_{T}\left(C \backslash \delta^{0}\right)=X_{T}(\partial \delta) \quad \text { and } \quad X_{T}(\delta \cup \gamma)=X_{T}(\delta)+X_{T}(\gamma) .
$$

(Throughout this paper $\delta^{0}$ and $\partial \delta$ denote the interior and the boundary of a set $\delta$ respectively.) The proof of the latter fact is similar to that of the Riesz decomposition theorem and uses the following identity:

$$
(\mu-T)^{-1} f_{x}(z)=(z-\mu)^{-1}\left[(\mu-T)^{-1} x-f_{x}(z)\right]
$$

for $\mu \notin \sigma(T)$.

(vi) An operator $T \in B(H)$ has the single-valued extension property if there ezists no nonzero $H$-valued analytic function $f$ such that $(z-T) f(z)$ $\equiv 0$. If $T$ has the single-valued extension property, so does its restriction to an invariant subspace. If $T$ has the single-valued extension property and $x \in H$ one may define

$$
\sigma_{T}(x)=\bigcap\left\{\delta: x \in X_{T}(\delta) \text { and } \delta \text { closed }\right\} .
$$

It is easy to see that $x \in X_{T}\left(\sigma_{T}(x)\right)$ and $X_{T}(\delta)=\left\{x: \sigma_{T}(x) \subseteq \delta\right\}$.

(vii) An invariant subspace $Y$ of $T$ is called a spectral maximal subspace of $T$ if $Z \subseteq Y$ for all invariant subspaces $Z$ of $T$ such that $o(T \mid Z)$ $\subseteq \mathcal{C}(T \mid Y)$. If $T$ has the single-valued extension property and $X_{T}(\delta)$ is closed, then $X_{T}(\delta)$ is a spectral maximal subspace of $T$ and $\sigma\left(T \mid X_{T}(\delta)\right) \subseteq$ $\delta \cap \sigma(T)[7$, p. 23$]$

(viii) Let $n \geq 2$ be a positive integer. An operator $T$ is called $n$-decomposable if for every open covering $G_{1}, G_{2}, \ldots, G_{n}$ of $\sigma(T)$ there exist spectral maximal subspaces $Y_{1}, Y_{2}, \ldots, Y_{n}$ of $T$ such that $H=Y_{1}+Y_{2}+\cdots+$ $Y_{n}$ and $\sigma\left(T \mid Y_{i}\right) \subseteq \bar{G}_{i}(i=1,2, \ldots, n)$. An operator is called decomposable if it is n-decomposable for all positive integers $n$ [7, p. 57].

(ix) Every normal operator is a spectral operator, and every spectral operator is decomposable. If $T$ is a spectral operator with the resolution of the identity $E$, then $X_{T}(\delta)=E(\delta) H$ for all closed sets $\delta[7, \mathrm{p} .33$ ].

(x) Every $n$-decomposable operator $T$ has the single-valued extension property and $X_{T}(\delta)$ is closed for all closed sets $\delta[14$, p. 215] $(n \geq 2)$. 
2. Main results. The main purpose of this section is to find some necessary and sufficient conditions for decomposability or normality of a cosubnormal operator (Theorems 1 and 3). Some of the results are extended to cosubspectral operators. Stampfli [20] shows that if $T$ is a hyponormal operator, then $X_{T}(\delta)$ is closed for all closed sets $\delta$, and if $T$ is cohyponormal, then there exists a closed set $\delta$ such that $X_{T}(\delta) \neq\{0\}$. In this direction we prove the following two lemmas.

Lemma 1. Let $A$ be a 2-decomposable operator defined on a Hilbert space $K$. Let $H$ be an invariant subspace of $A$ and let $S=A \mid H$. Then $X_{S}(\delta)$ is closed and $X_{S}(\delta) \subseteq H \cap X_{A}(\delta)$ for all closed sets $\delta$.

Proof. The fact that $X_{S}(\delta) \subseteq H \cap X_{A}(\delta)$ follows from the single-valued extension property for $A$. Now let $x_{n}$ be a Cauchy sequence in $x_{s}(\delta)$ converging to $x_{\text {. Let }} A_{\delta}=A \mid X_{A}(\delta)$. Since $A$ has the single-valued extension property, it follows that $\left(\lambda-A_{\delta}\right)^{-1} x_{n}$ has values in $H$ and converges uniformly to $\left(\lambda-A_{\delta}\right)^{-1} x$ on any compact subset of $C \backslash \delta$. Thus $x \in X_{s}(\delta)$ and hence $X_{S}(\delta)$ is closed.

Lemma 2. Let $N \in B(K)$ be an n-decomposable operator for some $n \geq 2$. Let $H$ be an invariant subspace of $N^{*}$. Let $Q: K \rightarrow K$ be the orthogonal projection onto $H$ and let $T=Q N Q \mid H$. Then $Q X_{N}(\delta) \subseteq X_{T}(\delta)$ for all closed sets $\delta$. Moreover, if $X_{T}\left(\delta_{n}\right)$ and $X_{T}\left(C \backslash \delta_{n}\right)$ are closed for a sequence $\left\{\delta_{n}\right\}$ of open sets forming a base for the topology of $\mathrm{C}$, then $T$ is n-decomposable and $T^{*}$ is 2-decomposable.

Proof. Let $x \in X_{N}(\delta)$ and let $N_{\delta}=N \mid X_{N}(\delta)$. Since $Q\left(\lambda-N_{\delta}\right)^{-1} x$ is analytic outside $\delta$ and $(\lambda-T) Q\left(\lambda-N_{\delta}\right)^{-1} x=x$ for $\lambda \notin \delta$, it follows that $Q x \in X_{T}(\delta)$ and thus $Q x_{N}(\delta) \subseteq X_{T}(\delta)$. Next let $G_{1}, G_{2}, \ldots, G_{n}$ be an open covering of $\sigma(T)$. Let $G_{n+1}$ be an open set such that $\bar{G}_{n+1} \cap \sigma(T)=\varnothing$ and $\sigma(N) \subseteq G_{1} \cup G_{2} \cup \cdots \cup G_{n+1}$. Let $x \in H$. We have $x=x_{1}+x_{2}+\cdots+x_{n}$ with $x_{i} \in X_{N}\left(\bar{G}_{i}\right), i=1,2, \ldots, n-1$, and $x_{n} \in X_{\underline{N}} \overline{\left(G_{n} \cup G_{n+1}\right)}$. Since $X_{B}(F)=X_{B}(F \cap \sigma(B))$, it follows that $Q x_{i} \in X_{T}\left(\bar{G}_{i}\right)(i=1,2, \ldots, n)$ and thus

$$
H=\sum_{1 \leq i \leq n} X_{T}\left(\bar{G}_{i}\right) .
$$

Now assume $X_{T}\left(\delta_{n}\right)$ and $X_{T}\left(C \backslash \delta_{n}\right)$ are closed, where $\left\{\delta_{n}\right\}$ is a sequence of open sets forming a base for the topology of $\mathrm{C}$. We claim $T$ has the single-valued extension property. Assume, if possible, that there exists a nonzero $H$-valued analytic function $f$ on some disc $\left|z-z_{0}\right|<r$ such that 
$(z-T) f(z) \equiv 0$. Let $f(z)=\Sigma a_{n}\left(z-z_{0}\right)^{n}$ and let $z_{0} \in \delta_{k} \subset \bar{\delta}_{k} \subset\left\{z:\left|z-z_{0}\right|\right.$

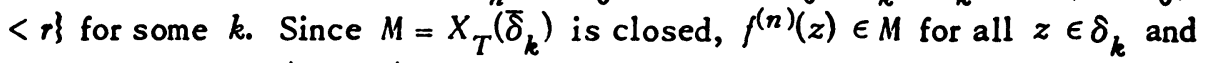
thus $f(z) \in M$ for $\left|z-z_{0}\right|<r$. Choose $z_{1}$ in the unbounded component of $C \backslash \bar{\delta}_{k}$ such that $\left|z_{1}-z_{0}\right|<r$, and $f\left(z_{1}\right) \neq 0$. It follows that there exists a $H$-valued analytic function $g$ on $C \backslash \delta_{k}$ with $(z-T) g(z) \equiv f\left(z_{1}\right)$. On the other hand $\left(z-z_{1}\right)^{-1} f\left(z_{1}\right)$ is a $H$-valued analytic function defined for $z \neq$ $z_{1}$ which agrees with $g(z)$ on the unbounded component of $C \backslash \sigma(T)$. Thus $g(z)=\left(z-z_{1}\right)^{-1} f\left(z_{1}\right)$ for $z$ in the unbounded component of $C \backslash \bar{\delta}_{k}$, a contradiction. Hence $T$ has the single-valued extension property.

Let $\delta$ be an arbitrary closed set. For each point $z \notin \delta$ there exists an integer $k(z)$ such that $z \in \delta_{k(z)} \subseteq \delta_{k(z)} \subseteq C \backslash \delta$. Since $T$ has the single-valued extension property, it follows that

$$
X_{T}(\delta)=\bigcap_{z \delta \delta} X_{T}\left(\mathbf{C} \backslash \delta_{k(z)}\right)
$$

and thus $X_{T}(\delta)$ is closed. Therefore, in view of $\$ 1$ (vii) and formula ( $\dagger$ ), $T$ is an $n$-decomposable operator. The last assertion follows from the fact that the adjoint of a 2-decomposable operator is 2-decomposable [10, p. 1057].

Remark 1. In Lemma 2, let $\delta$ be a closed set such that $\sigma(T) \cap \delta^{0} \neq \varnothing$. If $\sigma(N) \cap \delta^{0}=\varnothing$, then $\delta^{0} \subset \sigma_{p}(T)$ and thus $X_{T}(\delta) \neq\{0\}$. On the other hand, if $\sigma(N) \cap \delta^{0} \neq \varnothing$, then $X_{N}(\delta) \neq\{0\}$ and thus $Q X_{N}(\delta) \neq\{0\}$ [1, proof of Lemma 1.4]. Hence, again, $X_{T}(\delta) \neq\{0\}$.

Remark 2. The proof of Lemma 2 suggests the following proposition:

Let $T$ be an operator on some Banach space $Y$. Let $\delta_{n}$ be a sequence of open sets forming a base for the topology of C. If $X_{T}\left(\delta_{n}^{n}\right)^{n}$ is closed for all $n$, then $T$ has the single-valued extension property (cf. [2, Proposition 1.4]).

The following theorem contains a necessary and sufficient condition for decomposability of a cosubspectral operator.

Theorem 1. Let $N \in B(K)$ be a spectral operator, and let $H, T$, and $Q$ be as in Lemma 2. If $X_{T}(\partial \delta)$ is closed for some closed set $\delta$, then $X_{T}(\delta)$ and $X_{T}\left(C \backslash \delta^{0}\right)$ are closed, and $H=X_{T}(\delta)+X_{T}\left(C \backslash \delta^{0}\right)$. In particular if $X_{T}\left(\partial \delta_{n}\right)$ is closed for a sequence $\left\{\delta_{n}\right\}$ of open sets forming a base for the topology of $\mathrm{C}$, then $T$ is decomposable and $T^{*}$ is 2-decomposable.

Proof. Assume $X_{T}(\partial \delta)$ is closed. Let $x_{n}$ be a Cauchy sequence in $X_{T}(\delta)$ converging to $x$. Let $E$ be the resolution of the identity for $N$. Since $Q E(\mathrm{C} \backslash \delta) x_{n} \in X_{T}\left(\mathrm{C} \backslash \delta^{0}\right)$ and $x_{n}-Q E(\delta) x_{n} \in X_{T}(\delta)$ (Lemma 2), it follows that $Q E(C \backslash \delta) x_{n} \in X_{T}(\partial \delta)$ and thus $Q E(C \backslash \delta) x \in X_{T}(\partial \delta)$. Hence $x(=Q E(\delta) x$ 
$+Q E(C \backslash \delta) x)$ is in $X_{T}(\delta)$. This shows that $X_{T}(\delta)$ is closed. By a similar proof $X_{T}\left(\mathrm{C} \backslash \delta^{0}\right)$ is closed. Since $x=Q E(\delta) x+Q E(\mathbf{C} \backslash \delta) x$ for all $x \in H, H$ $=X_{T}(\delta)+X_{T}\left(C \backslash \delta^{0}\right)$. The rest of the proof follows from Lemma 2 .

In the following we write $H=M \oplus N$ if $M$ and $N$ are two (closed) subspaces of $H, M \cap N=\{0\}$, and $H=M+N$.

Lemma 3. Let $N \in B(K)$ be a spectral operator and let $H, T$, and $Q$ be as in Lemma 2. Let $E$ be the resolution of the identity for N. Assume $X_{T}(\partial \delta)=\{0\}$ for some closed set $\delta$. Then $H=X_{T}(\delta) \oplus X_{T}\left(C \backslash \delta^{0}\right)$ and $\|P\| \leq L$, where $P: H \rightarrow H$ is the projection onto $X_{T}(\delta)$ parallel to $X_{T}\left(C \backslash \delta^{0}\right)$ and $L=\sup \{\|E(\sigma)\|: \sigma$ Borel $\}$.

Proof. In view of Theorem $1, X_{T}(\delta)$ and $X_{T}\left(\mathrm{C} \backslash \delta^{0}\right)$ are closed, and $H=X_{T}(\delta)+X_{T}\left(\mathrm{C} \backslash \delta^{0}\right)$. Since $X_{T}(\delta) \cap X_{T}\left(\mathbf{C} \backslash \delta^{0}\right)=X_{T}(\partial \delta)=\{0\}, H=X_{T}(\delta)$ $\oplus X_{T}\left(\mathrm{C} \backslash \delta^{0}\right)$. Therefore $P$ is well defined and $P x=Q E(\delta) x$. This shows that $\|P\| \leq L$. Q.E.D.

If $T$ is a spectral operator on a separable Hilbert space and $\left\{C_{a}\right\}$ is a family of disjoint Jordan curves, then $X_{T}\left(C_{\alpha}\right)=\{0\}$ for all but a countable number of $a$. For a cosubspectral operator the following converse is true.

Theorem 2. Let $N, T, K, H$ and $Q$ be as in Lemma 3. Assume $X_{T}\left(\partial \delta_{n}\right)$ $=\{0\}$ for a sequence $\left\{\delta_{n}\right\}$ of open sets forming a base for the topology of $C$. Then $T$ is a spectral operator. Moreover if $N$ has an orthogonal resolution of the identity, so does $T$.

Proof. We use a "characterization" of spectral operators stated in Theorem XVI. 4.5 of $[8$, p. 2147].

Note first that since $T$ is decomposable (Theorem 1 ), $T$ has the singlevalued extension property and $X_{T}(\delta)$ is closed for all closed sets $\delta$. This proves conditions (A) and (C) of the "characterization".

Now we show that if $\delta$ is closed and $E(\delta)=0$, then $X_{T}(\delta)=\{0\}(E$ is the resolution of the identity for $N)$. Let $\left\{\sigma_{n}\right\}$ be the subsequence of $\left\{\delta_{n}\right\}$ consisting of all $\delta_{n}$ which lie entirely in $C \int^{2}$. Let $\gamma_{1}=\sigma_{1}$ and

$$
\gamma_{n}=\sigma_{n} \bigcup_{i<n} \sigma_{i} \quad(n=2,3, \ldots)
$$

Let $x \in X_{T}(\delta)$. We prove by induction that $Q E\left(\gamma_{n}\right) x=0(n=1,2, \ldots)$. Since $Q E\left(\gamma_{1}\right) x=Q E\left(\sigma_{1}\right) x=x-Q E\left(C \backslash \sigma_{1}\right) x \in X_{T}\left(\partial \sigma_{1}\right), Q E\left(\gamma_{1}\right) x=0$. Assume $Q E\left(\gamma_{i}\right) x=0$ for $i=1,2, \ldots, n-1$. It follows that

$$
Q E\left(\gamma_{n}\right) x=Q E\left(\gamma_{1} \cup \gamma_{2} \cup \cdots \cup \gamma_{n}\right) x
$$

and thus 


$$
Q E\left(\gamma_{n}\right) x \in X_{T}\left(\partial \gamma_{n} \cap \partial\left(\gamma_{1} \cup \cdots \cup \gamma_{n}\right)\right) \subseteq X_{T}\left(\partial \sigma_{n}\right)
$$

Hence $Q E\left(\gamma_{n}\right) x=0$. Therefore $x=Q E(\delta) x+\Sigma Q E\left(\gamma_{n}\right) x=0$ which implies that $X_{T}(\delta)=\{0\}$.

Let $\sigma$ and $\gamma$ be two disjoint closed sets. There exists a Cauchy domain $\delta$ such that (a) $\sigma \subset \delta$, (b) $\gamma \subset C \backslash \bar{\delta}$, and (c) $E(\partial \delta)=0$. It follows from Lemma 3 that

$$
\|x\| \leq L\|x+y\| \quad\left(x \in X_{T}(\sigma), y \in X_{T}(\gamma)\right),
$$

where $L=\sup \{\|E(\delta)\|: \delta$ Borel $\}$. This proves condition (B) of the "characterization".

Let $E$ be as above. Let $\delta$ be a closed set and let $\sigma_{n}$ be an increasing sequence of closed sets converging to $C \backslash \delta$. Since

$$
x=\lim \left[Q E(\delta) x+Q E\left(\delta_{n}\right) x\right]
$$

for all $x \in H$, it follows from Lemma 2 that every clqsed set $\delta$ is in the class $\delta_{1}(T)$ of all sets $\sigma$ with the property that vectors of the form $x+y$ with $\sigma_{T}(x) \subseteq \sigma$ and $\sigma_{T}(Y) \subseteq \mathrm{C} \backslash \sigma$ are dense in $H[8, \mathrm{p} .2138]$. Therefore to each closed set $\delta$ there corresponds a unique projection $F(\delta) \in B(H)$ such that $F(\delta) x=x$ if $\sigma_{T}(x) \subseteq \delta$ and $F(\delta) x=0$ if $\sigma_{T}(x) \subseteq C \backslash \delta[8$, p. 2138].

Now let $\delta$ and $\sigma_{n}$ be as above and assume moreover that $X_{T}(\partial \delta)=$ $X_{T}\left(\partial \sigma_{n}\right)=\{0\}(n=1,2, \ldots)$. Let $x \in H$. By the proof of Lemma $3, x=$ $\lim y_{n}$ and $\sigma\left(y_{n}\right) \subseteq\left(\delta \cup \sigma_{n}\right) \cap \sigma_{T}(x)$, where $y_{n}=Q E\left(\delta \cup \sigma_{n}\right) x(n=1,2, \ldots)$. Applying the Riesz decomposition theorem to $T \mid X_{T}\left(\delta \cup \sigma_{n}\right)$ yields $y_{n}=u_{n}$ $+v_{n}$, where $\sigma_{T}\left(u_{n}\right) \subseteq \delta \cap \sigma_{T}(x)$ and $\sigma_{T}\left(v_{n}\right) \subseteq \sigma_{n} \cap \sigma_{T}(x)(n=1,2, \ldots)$. This shows that every closed set $\delta$ with $X_{T}(\partial \delta)=\{0\}$ is in the class $\delta_{2}(T)$ of all sets $\sigma$ having the property that for every $x \in H$ and every $\epsilon>0$, there are vectors $x_{1}$ and $x_{2}$ with $\sigma_{T}\left(x_{1}\right) \subseteq \sigma_{T}(x) \cap \sigma, \sigma_{T}\left(x_{2}\right) \subseteq \sigma_{T}(x) \cap(C \backslash \sigma)$, and $\left\|x_{1}+x_{2}-x\right\|<\epsilon$.

Let $z_{0} \in \mathrm{C}, \in>0$, and let $x \in H$. Let $D_{r}=\left\{z:\left|z-z_{0}\right| \cdot<r\right\}$ for $r>0$. There exists a decreasing sequence $\{r(n)\}$ converging to a number $r(\infty)$ such that $0<r(\infty)<\epsilon$ and $X_{T}\left(\partial D_{r(n)}\right)=\{0\}(n=1,2, \ldots, \infty)$. Let $\delta=\bar{D}_{r(\infty)}$, $\sigma_{n}=C \backslash D_{r(n)}, \mathrm{y}_{n}=Q E\left(\delta \cup \sigma_{n}\right) x, u_{n}=F(\delta) y_{n}$, and let $v_{n}=F\left(\sigma_{n}\right) y_{n}$. It follows from the proof of Lemma 3 and the uniqueness of the set function $F$ on $\delta_{1}(T)$ that $y_{n}=F\left(\delta \cup \sigma_{n}\right) x$ and thus $u_{n}=F(\delta) x$ and $v_{n}=F\left(\sigma_{n}\right) x_{\text {. }}$ (Recall that the restriction of $F$ to $\delta_{2}(T)$ is a spectral measure [8, p. 2140].) Hence

$$
x=\lim \left[F(\delta)+F\left(\sigma_{n}\right)\right] x
$$


which implies that $\delta$ is in the class $\delta(T)$ of all sets $\sigma \in \mathcal{S}_{2}(T)$ for which there exist closed sets $\mu_{n}$ and $\nu_{n}$ in $\delta_{2}(T)$ with $\mu_{n} \subseteq \sigma, \nu_{n} \subseteq C \backslash \sigma, n=$ $1,2, \ldots$, and

$$
x=\lim \left[F\left(\nu_{n}\right)+F\left(\mu_{n}\right)\right] x \quad(x \in H) .
$$

Since $z_{0}$ and $\epsilon$ are arbitrary, it follows that every complex number is interior to a set of arbitrarily small diameter belonging to $S(T)$. This provescondition (D) of the "characterization" and with it the theorem.

Let $F_{s}(s \in \mathrm{R})$ be the resolution of the identity for a (bounded) Hermitian operator acting in a separable Hilbert space. There exist a family of Hilbert spaces $H_{s}(s \in \mathbf{R})$ such that the underlying Hilbert space is unitarily equivalent to $\int_{\mathrm{R}}^{\oplus_{s}^{s}} d \mu(s)$.

Moreover if an operator $T$ commutes with all projections $F_{s}$, then $T$ is unitarily equivalent to an operator of the form $\int^{\oplus} T_{s} d \mu(s)$, where $T_{s} \in B\left(H_{s}\right)$. (For the definitions and properties of direct integrals see [13, pp. 496-503].) Since $T$ is invertible if and only if $T_{s}$ is invertible a.e. $[d \mu]$, it follows that $\left(\lambda_{n}-T_{s}\right)^{-1}$ exists a.e. $[d \mu]$ simultaneously for all elements of a sequence $\left\{\lambda_{n}\right\}$ dense in $C \backslash \sigma(T)$. Thus $\sigma\left(T_{s}\right) \subseteq \sigma(T)$ a.e. $[d \mu]$.

In the following by a Jordan domain we mean an open set enclosed by a rectifiable Jordan curve. Theorem 2 can be sharpened for cosubnormal operators as follows.

Theorem 3. Let $N \in B(K)$ be a normal operator and let $T, H$, and $Q$ be as in Lemma 2. Let $\Delta$ be a totally ordered set and let $\left\{D_{a}\right\}_{a \in \Delta}$ be a fixed increasing chain of Jordan domains such that $X_{T}\left(\partial D_{a}\right)=\{0\}$ for all $\alpha \in \Delta$ and the area of the set

$$
\left.C\left(\Delta_{1}\right)=\left(\bigcap_{\beta \notin \Delta_{1}} \bar{D}_{\beta}\right)\right)\left(\bigcup_{\beta \in \Delta_{1}} D_{\beta}\right)
$$

is zero for any cut $\Delta_{1}$ in $\Delta$. (A subset $\Delta_{1}$ of $\Delta$ is a cut in $\Delta$ if any ele. ment in $\Delta_{1}$ is less than any element in the complement of $\Delta_{1}$ ) Then $T$ is a normal operator.

Proof. Assume without loss of generality that $H$ is separable and that $T$ has no nontrivial reducing invariant subspace on which it is normal. We claim $H=\{0\}$. Let $P_{a}$ be the projection onto $X_{T}\left(\bar{D}_{a}\right)$ parallel to $X_{T}\left(C \backslash D_{a}\right)$. Since $\left\|P_{a}\right\| \leq 1$, (Lemma 3 ), $\left\{P_{a}\right\}$ is an increasing sequence of orthogonal projections commuting with $T$.

Let $\pi$ be a chain of projections obtained from the completion of $\left\{P_{a}\right\}$. We claim $\pi$ has no gap. Assume, if possible, $\left(P^{-}, P^{+}\right)$is a gap in $\pi$. Let 
$\Delta_{1}=\left\{a \in \Delta: P_{a} \leq P^{-}\right\}$. Then $M=\left(P^{+}-P^{-}\right) H$ is a nontrivial reducing invariant subspace of $T$ and $\sigma(T \mid M) \subseteq \sigma\left(T \mid\left(P_{\beta}-P_{\alpha}\right) H\right) \subseteq \bar{D}_{\beta} \backslash D$ ar all $a \epsilon$ $\Delta_{1}$ and $\beta \in \Delta_{1}$. Thus the area of $\sigma(T \mid M)$ is zero and hence $T \mid M$ is a normal operator, a contradiction [16]. Therefore there exists a (strictly increasing) resolution of the identity $F_{s}(0 \leq s \leq 1)$ (belonging to a Hermitian operator) whose range coincides with $\pi$ [5, Theorem 18.1]. Thus (up to unitary equivalence):

$$
H=\int_{[0,1]}^{\oplus} H_{s} d \mu(s) \text { and } T=\int_{[0,1]}^{\oplus} T_{s} d \mu(s),
$$

where $T_{s}$ is cohyponormal a.e. [ $d \mu$ ]. (Actually Bastian [3] shows that $T_{s}$ is cosubnormal a.e. $[d \mu]$.)

For $[a, b] \subseteq[0,1]$ let

$$
T_{[a, b]}=\int_{[a, b]}^{\oplus} T_{s} d \mu(s) \text { and } H_{[a, b]}=\int_{[a, b]}^{\oplus} H_{s} d \mu(s) \text {. }
$$

It is easy to see that $H_{[a, b]}=\left(F_{b}-F_{a}\right) H$ and $T_{[a, b]}=T \mid H_{[a, b]}$. Let $\delta(n, k)=[(k-1) / n, k / n]$ for $k=1,2, \ldots, n$, and $n=1,2, \ldots$. Since

$$
\mu\left(\left\{s \in \delta(n, k): \sigma\left(T_{s}\right) \not \subset \sigma\left(T_{\delta(n, k)}\right)\right\}\right)=0
$$

for all $\delta(n, k)$, it follows that

$$
\sigma\left(T_{s}\right) \subseteq \bigcap_{(n, k) \in \Gamma(s)} \sigma\left(T_{\delta(n, k)}\right)
$$

a.e. $[d \mu]$, where $\Gamma(s)=\{(n, k): s \in \delta(n, k)\}$. Let $s$ satisfy (*). Let $\Delta_{1}=$ $\left\{a \in \Delta: P_{a}<F_{s}\right\}, \Delta_{2}=\left\{\alpha \in \Delta: P_{\alpha}=F_{s}\right\}$, and $\Delta_{3}=\Delta \backslash\left(\Delta_{1} \cup \Delta_{2}\right)$. Since $P_{a}$ is constant on $\Delta_{2}$, it follows that

$$
o(T) \subseteq\left(\bigcap_{a \in \Delta_{2}} \bar{D}_{\alpha}\right) \cup\left(\bigcap_{\alpha \in \Delta_{2}}\left(C \backslash D_{\alpha}\right)\right),
$$

and thus $\sigma\left(T_{s}\right) \subseteq C\left(\Delta_{1}\right) \cup C\left(\Delta_{1} \cup \Delta_{2}\right)$. Hence the area of $o\left(T_{s}\right)=0$. This shows that $T_{s}$ is normal a.e. $[d \mu]$. Therefore $T$ is normal and thus $H=$ \{0\}. The proof of the theorem is complete.

Definition. An operator $T$ is said to satisfy a boundedness condition (B) if there exists a positive constant $L$ such that $\|x\| \leq L\|x+y\|$ for all $x \in X_{T}(\delta), y \in X_{T}(\sigma)$, and all pairs of disjoint closed sets $\delta$ and $\sigma$. (We do not impose the single-valued extension property on $T[8, \mathrm{p} .2138]$ )

Stampfli [20] shows that a cohynormal operator satisfying a boundedness condition (B) has a nontrivial invariant subspace. The following theorem shows that such cosubnormal (resp. cosubspectral) operators are indeed normal (resp. spectral). 
Theorem 4. A cosubnormal (resp. cosubspectral) operator $T \in B(H)$ satisfying a boundedness condition (B) is normal (resp. spectral).

Proof. Assume without loss of generality that $H$ is separable. Let $N \in B(K)$ be the adjoint of a normal (resp. spectral) extension of $T^{*}$ and let $K$ be separable. Let $E$ be the resolution of the identity for $N$. Let $\left\{C_{a}\right\}$ be an arbitrary family of disjoint rectifiable Jordan curves. Since $K$ is separable, $E\left(C_{\alpha}\right)=0$ for all but a countable number of $\alpha_{\text {. Let } \delta \text { be a }}$ closed set such that $E(\delta)=0$. Let $G_{n}$ be a decreasing sequence of open sets converging to $\delta$. The sequence $E\left(G_{n}\right)$ converges strongly to zero as $n \rightarrow \infty$. Let $x \in X_{T}(\delta)$. It follows from the boundedness condition (B) and Lemma 2, that $\|x\| \leq L\left\|x-Q E\left(C \backslash G_{n}\right) x\right\|$ for all $n$. Letting $n \rightarrow \infty$ yields $x=0$. Thus $X_{T}(\delta)=\{0\}$ and hence, in view of Theorem 3 (resp. Theorem 2), $T$ is a normal (resp. spectral) operator.

3. Eigenvalues of cosubnormal operators. Let $\sigma_{p}(T)$ be the set of all eigenvalues of an operator $T \in B(H)$. Let $\sigma_{p \perp}(T)$ be the set of all eigenvalues $\lambda$ of $T$ such that the null space $N(\lambda-T)$ reduces $T$. Let $\sigma_{p 0}(T)$ be the set of all complex numbers $\lambda$ such that $\lambda$ is in the domain of some nonzero $H$-valued analytic function $f(z)$ which has a connected domain and satisfies $(z-T) f(z) \equiv 0$. It is true that $\sigma_{p 0}(T) \subseteq \sigma_{p}(T)[7$, p. 22] and $\sigma_{p 0}(T) \cap \sigma_{p_{\perp}}(T)=\varnothing$. (Because if $\lambda \in \sigma_{p_{\perp}}(T)$ and $\lambda$ is in the domain of an analytic function $f$ satisfying $(z-T) f(z) \equiv 0$, then $f(z) \perp N(\lambda-T)$ for all $z$ and $\lambda \in \sigma_{p}\left(T \mid N(\lambda-T)^{\perp}\right)$, a contradiction.) Also if $S$ is the restriction of an operator $N \in B(K)$ to an invariant subspace $H$ (of $N$ ), then

$$
\sigma\left(S^{*}\right) \backslash \sigma\left(N^{*}\right) \subseteq \sigma_{p 0}\left(S^{*}\right) \text {. }
$$

(Let $Q$ be the projection on to $H$ and let $\lambda$ and $\mu$ be two points of $\sigma\left(s^{*}\right)$ lying in the same component $G$ of $C \backslash \sigma\left(N^{*}\right)$. Let $x$ be a nonzero vector in $H$ such that $(\mu-T) x=0$. Then $f(z)=(z-\mu)^{-1} x-Q\left(z-N^{*}\right)^{-1} x \quad(z \in G \backslash\{\mu\})$ is a nonzero analytic function having $\lambda$ in its (connected) domain and satisfying $(z-T) f(z) \equiv 0$.$) (In view of the Wold decomposition theorem for isometry operators,$ formula $(* *)$ provides another proof for Lemma 1.7 of $[7$, p. 10].)

The following lemmas study the relation between $\sigma_{p}(T)$ and the geometrical shape of $\sigma(T)$ for a cosubnormal or cohyponormal operator $T$.

Lemma 4. Let $T$ be a cohynormal operator. Let $\lambda \in \partial \sigma(T)$. Assume there exists a constant $K$ and a sequence $\left\{\lambda_{n}\right\}$ in $C \backslash \sigma(T)$ such that $\lim \lambda_{n}$ $=\lambda$ and $\left|\lambda-\lambda_{n}\right| \leq K \operatorname{dist}\left(\lambda_{n}, \sigma(T)\right)$ for $n=1,2, \ldots$. Then $\lambda \in \sigma_{p \perp}(T)$ if $\lambda \in \sigma_{p}(T)$.

Proof. Assume without loss of generality that $N\left(\lambda-T^{*}\right)=\{0\}$. We claim 
$N(\lambda-T)=\{0\}$. By $[18$, p. 469]

$$
\left\|\left(\lambda_{n}-T\right)^{-1}\right\| \leq 1 / \operatorname{dist}\left(\lambda_{n}, \sigma(T)\right) \leq K /\left|\lambda-\lambda_{n}\right| \text { for } n=1,2, \ldots \text {. }
$$

Therefore $H=N(\lambda-T) \oplus \vec{R}(\lambda-T)[12, \mathrm{p} .62]$. (Here $\bar{R}$ denotes the closure of the range.) Since $N\left(\overline{-}-T^{*}\right)=\{0\}, \bar{R}(\lambda-T)=H$ and thus $N(\lambda-T)=\{0\}$.

(For special cases of Lemma 4 see $[15]$ and $[19$, p. 135].)

Theorem 5. Let $E$ be a compact subset of the plane. Let $\mathbb{Q}$ be a fam. ily of analytic functions having $E$ in their domains. Let $H$ be the span of $\mathcal{Q}$ in $L^{2}(E, d x d y)$. Let $S$ be the multiplication by $z$ in $H$ and let $T=S^{*}$. Then

(a) $X_{s}(\delta)=\{0\}$ for all closed subsets $\delta$ of $E^{0}$,

(b) $\left(\stackrel{E}{E}^{0}\right) * \subseteq \sigma_{p 0}(T)$,

where $\Delta^{*}=\{\lambda: \lambda \in \Delta\}$. In particular $S$ and $T$ are not 2-decomposable if $E^{0}$ $\neq \varnothing$.

Proof. By the area mean value theorem the elements of $H$ are analytic in $E^{0}$. Thus if $f \in X_{S}(\delta)$, it follows from Lemma 1 that $f(z)=0$ for all $z \notin$ $\delta$ and thus $f \equiv 0$ on $E$. This proves (a).

Let $\lambda$ be the center of a disc $|z-\lambda|<r$ lying entirely on $E^{0}$. We can assume without loss of generality that $\lambda=0$ and $r=1$. Let $V$ be the bilateral weighted shift $V e_{n}=[(n+1) /(n+2)]^{1 / 2} e_{n+1}$ for $n \geq 0$ and $V e_{n}=$ $e_{n+1}$ for $n<0$ defined on some Hilbert space $K_{1}$. Let $W$ be the multiplication by $z$ in $K_{2}=L^{2}(E \backslash D, d x d y)$, where $D$ is the unit disc. Let $K=K_{1}$ $\oplus K_{2}$ and $N=W \oplus V$. It is easy to see that $\sigma(N) \cap D=\varnothing$. In view of [11, Problem 25] the mapping $U: H \rightarrow K$ defined by

$$
U f=(f \mid E \backslash D) \oplus \sum_{n=0}^{\infty} \frac{f^{(n)}(0)}{n !}[\pi /(n+1)]^{1 / 2} e_{n}
$$

is an isometry and $U S=N U$. Therefore $S$ is unitarily equivalent to a part of $N$. Since $D \subseteq \sigma(S)$, it follows from (**) that $D \subseteq \sigma_{p 0}(T)$. Statement (b) is proved.

The last assertion follows from the fact that $T$ does not have the singlevalued extension property. The proof of the theorem is complete.

For a compact set $X$ and a (positive) measure $\mu$ on $X$, let $C(X), R(X)$, $R(X)$, and $R^{2}(X, d \mu)$ denote the continuous functions on $X$, the rational functions with poles off $X$, the uniform closure of $R(X)$, and the closure of $R(X)$ in $L^{2}(X, d \mu)$, respectively.

Theorem 6. Let $X$ be a compact subset of $C$ such that, for any open disc $D, X \cap D \neq \varnothing$ implies $R(X \cap \bar{D}) \neq C(X \cap \bar{D})$. Then there exists a com- 
pletely nonnormal cosubnormal operator $T$ such that

$$
\overline{\sigma_{p}(T)}=\sigma(T)=X .
$$

(An operator is called completely nonnormal, if it has no nonzero reducing invariant subspaces on which it is normal.)

Proof. In view of Theorem 5, we can assume without loss of generality that $X^{0}=\varnothing$. Let $Y=X^{*}$. Following the argument in [6, p. 242] we can find a sequence $\left\{\lambda_{n}\right\}$ dense in $Y$ and a sequence of Borel probability measures $\left\{\mu_{n}\right\}$ such that

$$
f\left(\lambda_{n}\right)=\int_{Y} f d \mu_{n} \quad(f \in R(Y))
$$

and $\mu_{n}(\{\lambda\})<1$. By replacing $\mu_{n}$ by $\left[\mu_{n}-\mu_{n}\left(\left\{\lambda_{n}\right\}\right)\right] /\left[1-\mu_{n}\left(\left\{\lambda_{n}\right\}\right)\right]$, we can assume without loss of generality that $\mu_{n}\left(\left\{\lambda_{n}\right\}\right)=0$. Let $A_{n}$ be the multiplication by $z$ in $R^{2}\left(Y, d \mu_{n}\right)$. It follows from $(* * *)$ and the Schwarz inequality that the nonzero linear functional $f \rightarrow f\left(\lambda_{n}\right), f \in R(Y)$, has a bounded extension to $R^{2}\left(Y, d \mu_{n}\right)(n=1,2, \ldots)$. Therefore the range of $\lambda_{n}-A_{n}$ lies in a closed subspace of codimension 1 of $R^{2}\left(Y, d \mu_{n}\right)$, and hence ${ }^{n} \lambda_{n} \in \sigma_{p}\left(\left(A_{n}\right) *\right)$ $(n=1,2, \ldots)$. Obviously $\lambda_{n}$ is not an eigenvalue of $A_{n}$, because $\mu_{n}\left(\left\{\lambda_{n}\right\}\right)$ $=0$. Thus $A_{n}$ is a nonnormal subnormal operator. Let $B_{n}$ be the completely nonnormal part of $A_{n}$. It follows that $\lambda_{n} \in \sigma\left(B_{n}\right) \subseteq \sigma\left(A_{n}\right) \subseteq Y$. Let

$$
S=\Sigma \oplus B_{n} \text { and } T=S^{*} \text {. }
$$

The operator $T$ satisfies all the requirements of the theorem.

Remark 3. Brennan [4, Pp. 314-315] constructs a Swiss cheese $E$ with the following properties:

(a) the linear functional $f \rightarrow f(\lambda)(f \in R(E))$ has a bounded extension to $R^{2}(E, d x d y)$ for almost every point $\lambda$ in $E$ (such points $\lambda$ are called bounded point evaluations of $\left.R^{2}(E, d x d y)\right)$,

(b) whenever two functions in $R^{2}(E, d x d y)$ coincide on a set of positive area in $E$, they coincide a.e. [dxdy].

Let $E$ be such a set and let $S$ be the multiplication by $z$ in $R^{2}(E, d x d y)$. Let $T=S^{*}$. It follows that $\sigma_{p 0}(T)=\sigma_{p \perp}(T)=\varnothing$, and the area of $E^{*} \backslash \sigma_{p}(T)$ is zero. (Note that, in view of Lemma 4, there are points in $\sigma(T)$ which are not eigenvalues of $T_{\text {.) }}$ Let $G_{1}$ and $G_{2}$ be two open sets such that

(i) $\sigma(S) \subseteq G_{1} \cup G_{2}$,

(ii) the sets $E \backslash \bar{G}_{i}(i=1,2)$ have positive areas. Let $f_{i} \in X_{T}\left(\bar{G}_{i}\right)$ $(i=1,2)$. By Lemma $1, f_{i}=0$ on $E \backslash \bar{G}_{i}$ and thus $f_{i}=0$ on $E(i=1,2)$. Thus $S$ (and hence $T$ ) is not 2-decomposable, though it has a nowhere dense spectrum. 
One may raise the following question.

Question 1. Is there a nonnormal 2-decomposable subnormal operator?

In view of Theorem 3, a negative answer to the following question will provide a negative answer to Question 1.

Question 2. Is there a decomposable operator $T \in B(H)$ such that $X_{T}\left(C_{a}\right)$ $\neq\{0\}$ for an uncountable number of disjoint (piecewise smooth) Jordan curves $C_{a}$ ?

Remark 4. The proof of Theorem 6 contains a negative answer to a question raised by Putnam in [15, p. 282].

Let $X$ be a compact set. A point $x \in X$ is called a peak point of $R(X)$ if there exists a function $f \in R(X)$ such that $f(x)=1$ and $f(y)<1$ for all $y \in X \backslash\{x\}$. (Such a function $f$ is said to peak at $x_{0}$ ) Let $p(X)$ denote the set of all peak points of $R(X)$. We prove the following theorem.

Theorem 7. If $T \in B(H)$ is a cosubnormal operator, then $p(\sigma(T)) \cap \sigma_{p}(T)$ $\subseteq \sigma_{p_{\perp}}(T)$.

Proof. Let $\lambda \in p(\sigma(T)) \cap \sigma_{p}(T)$. We may and shall assume without loss of generality that $\lambda=0$. Let $S=T^{*}, A$ be the minimal normal extension of $S$, and let $E$ be the resolution of the identity for $A$. Let $x$ be a unit vector such that $T x=0$. We prove $S x=0$. Since $(S y \mid x)=0$ for all $y \in H,(g(A) x \mid x)$ $=(g(S) x \mid x)=g(0)$ for all $g \in R(\sigma(S))$. Thus $(g(A) x \mid x)=g(0)$ for all $g \in$ $R(\sigma(S))$. Hence if $f \in R(\sigma(S))$ and $f$ peaks at 0 , then $\left(f^{n}(A) x \mid x\right)=1$ for $n$ $=1,2, \ldots$. (Note that $0 \in p(\sigma(S))$.) Therefore by dominated convergence theorem

$$
\begin{aligned}
1 & =\lim \left(f^{n}(A) x \mid x\right)=\lim \int f^{n} d\|E x\|^{2} \\
& =\int \lim f^{n} d\|E x\|^{2}=\|E(\{0\}) x\|^{2} .
\end{aligned}
$$

Thus $A x=0$ and consequently $S x=0$. It follows that $N(S) \supseteq N(T) \supseteq N(S)$ which completes the proof of the theorem.

Note. In view of Proposition 3.6 and Theorem 6.1 of [22, pp. 13 and 45],

$$
\sigma_{p}(T) \backslash \sigma_{p_{\perp}}(T) \subseteq \sigma(T) \backslash \bigcup \partial G_{i}
$$

where $T$ is a cosubnormal operator and $\left\{G_{i}\right\}$ is the class of all components of $\mathrm{C} \backslash \sigma(T)$.

\section{REFERENCES}

1. C. Apostol, Restrictions and quotients of decomposable operators in a Banach space, Rev. Roumaine Math. Pures Appl. 13 (1968), 147-150. MR 37 \#6786.

2. - Roots of decomposable operator-valued analytic functions, Rev. Roumaine Math. Pures Appl. 13 (1968), 433-438. MR 38 \#1548. 
3. J. Bastian, Dissertation, Indiana University, 1973.

4. J. E. Brennan, Approximation in the mean and quasianaliticity, J. Functional Analysis 12 (1973), 307-320.

5. M. S. Brodskiī, Triangular and Jordan representations of linear operators, "Nauka", Moscow, 1969; English transl., Transl. Math. Monographs, vol 32, Amer. Math. Soc., Providence, R. I., 1971. MR 41 \#4283.

6. K. F. Clancey and C. R. Putnam, The local spectral behavior of completely subnormal operators, Trans. Amer. Math. Soc. 163 (1972), 239-244. MR 45 \#934.

7. I. Colojoară and C. Foiaș, The theory of generalized spectral operators, Gordon \& Breach, New York, 1968.

8. N. Dunford and J. T. Schwartz, Linear operators. III: Spectral operators, Interscience, New York, 1971.

9. C. Foias, Some applications of spectral sets. I. Harmonic-spectral measure, Akad. R. P. Romîne. Stud. Cerc. Mat. 10 (1959), 365-401; English transl., Amer. Math. Soc. Transl. (2) 61 (1967), 25-62. MR 22 \#8340.

10. S. Frunză, A duality theorem for decomposable operators, Rev. Roumaine Math. Pures Appl. 16 (1971), 1055-1058. MR $46 \# 710$.

11. P. R. Halmos, A Hilbert space problem book, Van Nostrand, Princeton, N. J., 1967. MR $34 \# 8178$.

12. A. S. Markus, Some criteria for the completeness of a system of root vectors of a linear operator in a Ban ach space, Mat. Sb. 70 (112) (1966), 526-561; English transl., Amer. Math. Soc. Transl. (2) 85 (1969), 51-91. MR 35 \#7151.

13. M. A. Naimark, Normed rings, GITTL, Moscow, 1956; English transl., Noordhoff, Groningen, 1959. MR 19, 870; $22 \# 1824$.

14. S. Plafker, On decomposable operators, Proc. Amer. Math. Soc. 24 (1970), 215-216. MR $40 \# 1804$.

15. C. R. Putnam, Eigenvalues and boundary spectra, Illinois J. Math. 12 (1968), 278-282. MR $37 \# 2030$.

16. - An inequality for the area of hyponormal spectra, Math. Z. 116 (1970), 323-3.30. MR $42 \# 5085$.

17. - Resolvent vectors, invariant subspaces, and sets of zero capacity, Math. Ann. 205 (1973), 165-171. MR $48 \# 4772$.

18. J. G. Stampfli, Hyponomal operators and spectral density, Trans. Amer. Math. Soc. 117 (1965), 469-476; errata, ibid., 115 (1965), 550. MR 30 \#3375; 33 \#4686.

19. - A local spectral theory for operators. III: Resolvents, spectral sets and similarity, Trans. Amer. Math. Soc. 168 (1972), 133-151. MR 45 \#182.

20. - Spectral subspaces of hyponormal operators (to appear).

21. A. E. Taylor, Introduction to functional analysis, Wiley, New York; Chapman \& Hall, London, 1958. MR 20 \#5411.

22. L. Zalcman, Analytic capacity and rational approximation, Lecture Notes in Math., no. 50, Springer-Verlag, Berlin and New York, 1968. MR 37 \#3018.

DEPARTMENT OF MATHEMATICS, DALHOUSIE UNIVERSITY, HALIFAX, NOVA SCOTIA, CANADA 